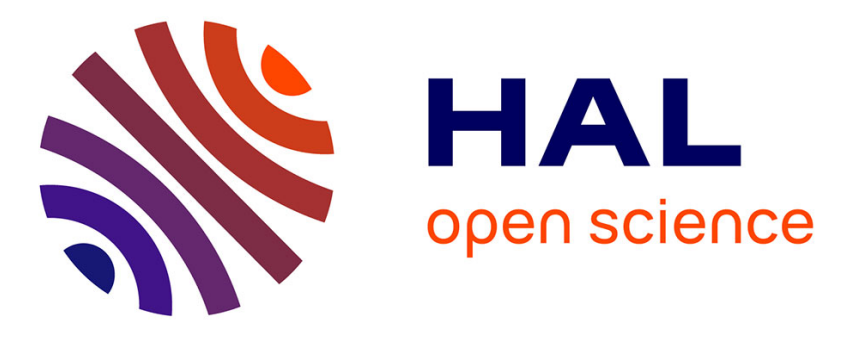

\title{
Dual magnetic effects on soot production in partially premixed flames
}

\author{
A Jocher, J Bonnety, H Pitsch, T Gomez, Guillaume Legros
}

\section{To cite this version:}

A Jocher, J Bonnety, H Pitsch, T Gomez, Guillaume Legros. Dual magnetic effects on soot production in partially premixed flames . Proceedings of the Combustion Institute, 2016, 36, pp.1377-1385. 10.1016/j.proci.2016.05.017 . hal-01433363

\section{HAL Id: hal-01433363 https://hal.sorbonne-universite.fr/hal-01433363}

Submitted on 12 Jan 2017

HAL is a multi-disciplinary open access archive for the deposit and dissemination of scientific research documents, whether they are published or not. The documents may come from teaching and research institutions in France or abroad, or from public or private research centers.
L'archive ouverte pluridisciplinaire HAL, est destinée au dépôt et à la diffusion de documents scientifiques de niveau recherche, publiés ou non, émanant des établissements d'enseignement et de recherche français ou étrangers, des laboratoires publics ou privés. 


\title{
Dual magnetic effects on soot production in partially premixed flames
}

\author{
A. Jocher ${ }^{\mathrm{a}}$, J. Bonnety ${ }^{\mathrm{a}}$, H. Pitsch ${ }^{\mathrm{b}}$, T. Gomez ${ }^{\mathrm{c}}$, G. Legros ${ }^{*, a}$ \\ ${ }^{a}$ Sorbonne Universités, UPMC Univ Paris 06, CNRS, UMR 7190 Institut Jean le Rond \\ d'Alembert, F-75005 Paris, France \\ ${ }^{b}$ RWTH Aachen University, Institute for Combustion Technology, Aachen, Germany \\ ${ }^{c}$ Université Lille I, Lille, France
}

\begin{abstract}
The present paper further explores the potential of the gradient of the square of the magnetic flux density $\left(\nabla\left(\mathbf{B}^{2}\right)\right)$ to control the soot production in flames. Experimental investigations assess for the first time the influence of $\nabla\left(\mathbf{B}^{2}\right)$ on the soot production in laminar axisymmetric partially premixed flames. The steady rich ethylene/oxygen flames are established in different coflowing mixtures, composed of oxygen and nitrogen, over a Santoro type burner, which is located in an electromagnet. The flame experiences different magnitudes of upward $\nabla\left(\mathbf{B}^{2}\right)$, ranging from 0 to $18.2 \mathrm{~T}^{2} / \mathrm{m}$, as well as different oxygen contents of the coflow, ranging from $21 \%$ to $50 \%$ in volume, and different levels of the equivalence ratio, ranging from 5 to $\infty$. Soot temperature and volume fraction are mapped in the flame by a Modulated Absorption/Emission technique. Increasing the magnitude of $\nabla\left(\mathbf{B}^{2}\right)$ allows for the modification of soot production in the flame. A reversal of the magnetic effect compared to the non-premixed case is observed. For non-premixed flames, the upward $\nabla\left(\mathbf{B}^{2}\right)$ systematically leads to an increase in soot production, while this magnetic gradient can induce an overall reduction in soot production in some partially
\end{abstract}


premixed flames. This original reversal is attributed to a switch of the magnetic force direction. As a result, this supplementary force can induce an increase or a decrease of the residence time in the rich region of a partially premixed flame, depending mainly on the fields of oxygen mole fraction and temperature in this region. In conjunction with more elaborated magnetic fields, these opposite trends may contribute to strategies for the control of soot production.

Keywords: Sooting tendency, laminar partially premixed flame, magnetic

field 


\section{Introduction}

The presence of particulate matter, especially fine-particulate soot, in urban air is now associated with major health hazards, such as cardiac arrest and pulmonary diseases, therefore higher mortality rates $[1,2]$.

Post-treatment processes, such as filters [3] and catalysts [4], have been developed to mitigate soot release in the exhaust of combustion devices. Additionally, both promising and complementary in-situ strategies aim to control soot production in the combustion zones. In that regard, the design of new fuels [5], the addition of exhaust gases to the combustion process [6], and the combustion control by electrical fields [7] has led to some success.

Recently, Jocher et al. [8] conducted original experiments evidencing for the first time the influence of the gradient of the square of the magnetic flux density $\left(\nabla\left(\mathbf{B}^{2}\right)\right)$ on soot production in a laminar axisymmetric non-premixed coflow flame. Soot volume fraction in the flame was mapped by a Laser Extinction Measurement technique. These authors showed that increasing the magnitude of the upward $\nabla\left(\mathbf{B}^{2}\right)$ allows for the modification of soot produc-

tion in the flame due to the relatively high paramagnetic susceptibility of oxygen. Furthermore, this modification was shown to enable the shift among similar soot concentration profiles in the flame, just as the variation of oxygen content in the coflow can do.

To further explore the potential of such a soot production control, the present paper focuses on the influence of an upward $\nabla\left(\mathbf{B}^{2}\right)$ on the soot production in partially premixed flames. Indeed, turbulent mixing may produce locally partially premixed conditions in practical non-premixed combustion system, such as staged combustors and spray flames. As a result, some 
studies investigated soot formation and oxidation in academic laminar axisymmetric partially premixed coflow flames $[9,10,11]$.

For the present study, the experimental setup implemented by Jocher et al. [8] is used. In addition, fields of both soot temperature and volume fraction are provided by the Modulated Absorption/Emission (MAE) technique that Legros et al. [12] have recently developed for laminar axisymmetric flames. These authors emphasized that this self-calibrating technique inherently accounts for the soot self-absorption of the signals to be processed. Furthermore, the retrieval of the temperature field does not depend on the selection of the soot refractive index, since the absorption coefficient field is determined through laser attenuation measurements. On the contrary, the determination of soot temperature by any conventional multi-wavelength pyrometry requires a model for the spectral dependence of the soot refractive index $[13,14]$, a quantity that is a topic of ongoing debate as large discrepancies are reported in the literature $[15,16]$.

\section{Experimental configuration and procedure}

Figure 1 displays the schematic of the original experimental setup that allows soot temperature and volume fraction to be mapped simultaneously in partially premixed flames experiencing magnetic fields.

\subsection{Flames}

The partially premixed flames are established at constant room temperature of $295 \mathrm{~K}$ over an atmospheric axisymmetric coflow burner, identical to the one described by Santoro et al. [17]. With this burner configuration, the 
sooting behaviours of a wide range of fuels, in particular ethylene, have been extensively documented in the literature $[18,19]$.

The fuel mixture flows through the vertical axial brass duct, which has an effective diameter of injection $d_{\mathrm{F}}$ of $11 \mathrm{~mm}$. This mixture is composed of ethylene and oxygen. Two Bronkhorst EL-FLOW mass flow controllers enable the variation of both the oxygen and ethylene flow rates, therefore the equivalence ratio $\phi$ of the fuel mixture.

The coflow mixture is introduced into a concentric brass cylinder of 102 mm inner diameter. This stream consists of an oxygen/nitrogen mixture. As nitrogen is the the major inert component in air, it is selected as the balance gas. Two other Bronkhorst EL-FLOW mass flow controllers allow both the oxygen and nitrogen flow rates to be adjusted. Doing so, the oxygen molar content $\mathrm{X}_{\mathrm{O}_{2}, \mathrm{c}}$ of the coflow stream can be varied.

All gases come from high-purity gas cylinders (99.9\% stated purities). Further details about the characteristics of the present burner can be found in earlier publications $[20,21,8]$.

In the following, the vertical burner's axis of symmetry is referred to as $\mathrm{O} z$ and its origin is located at the nozzle exit plane, defining the height above the burner (HAB). The cross-stream coordinate is $r$, which is the distance from the axis of symmetry.

For the present study, the flow rates of both coflow and fuel mixture streams are kept constant to ensure similar hydrodynamic conditions at the burner inlet. The fuel mixture flow rate is set to $3.5 \pm 0.07 \mathrm{~cm}^{3} / \mathrm{s}$, corresponding to a mean injection velocity of $\mathrm{V}_{\mathrm{F}}=3.98 \pm 0.09 \mathrm{~cm} / \mathrm{s}$. The flow rate of the coflow stream is set to $717 \mathrm{~cm}^{3} / \mathrm{s}$, corresponding to a mean coflow 
velocity of $\mathrm{V}_{\mathrm{c}}=8.9 \pm 0.09 \mathrm{~cm} / \mathrm{s}$. These flow rates are chosen so that the flames presented in this study are contained within the domain of almost uniform $\nabla\left(\mathbf{B}^{2}\right)$. These hydrodynamic conditions are also very similar to those established for the study of sooting non-premixed flames experiencing high magnetic fields [8]. Furthermore, all flames are laminar and steady.

The study consists of investigating partially premixed flames for three different equivalence ratios of the fuel mixture, i.e. $\phi=5,6.8$, and $\infty$. To this end, the ethylene flow rate is adjusted to $2.19,2.43$, and $3.5 \mathrm{~cm}^{3} / \mathrm{s}$, respectively, while oxygen is added to obtain the constant fuel mixture flow rate. These flames burn in oxidizer streams of three different $\mathrm{O}_{2}$ contents, i.e. $\mathrm{X}_{\mathrm{O}_{2}, \mathrm{c}}=0.3,0.4$ and 0.5 . Every flame experiences the three levels of upward $\nabla\left(\mathbf{B}^{2}\right)$ reported by Jocher et al. [8], i.e. $1.8,7.1$, and $18.2 \mathrm{~T}^{2} / \mathrm{m}$. These are referred as $\mathrm{MagF}$ 1, MagF 2, and $\mathrm{MagF}$ 3, respectively. The absence of magnetic field is called MagF 0.

\subsection{Electromagnet}

The electromagnet shown in Fig.1 consists of two identical coils of inner radius $\mathrm{R}=120 \mathrm{~mm}$ facing each other. Both coils have a common horizontal axis and are separated by a distance similar to R. A power supply delivers an adjustable DC electrical current, I, that flows through the electric coils, which creates the magnetic field. Such a configuration increases the maximum magnetic field magnitude that a single coil can generate and promotes the field uniformity within the system core. An iron magnetic circuit then confines the magnetic field lines.

A two-dimensional numerical simulation has been carried out to compute 
the magneto static field. A set of measurements by a Hall effect sensor enable the calibration of the computed fields. Details of this procedure are given in Ref.[20].

In order to expose the flames to fairly uniform upward $\nabla\left(\mathbf{B}^{2}\right)$, the burner tip is located $130 \mathrm{~mm}$ below the coils' axis of symmetry [8]. The horizontal component magnitudes of the gradients were checked to be negligible at this location. Therefore, the direction of $\nabla\left(\mathbf{B}^{2}\right)$ and the burner's axis of symmetry are identical.

None of the rig components is magnetic to avoid mechanical interferences. Furthermore, as shown in Ref. [20], reversing the current in the coils does not lead to any flame deviation, even when moving the camera line-ofsight around the burner axis. Therefore, the Lorentz force acting on moving charged species or particles in the flame can be considered negligible.

\subsection{Diagnostics and data processing}

The optical arrangement that enables the MAE technique is shown in Fig.1. This technique provides simultaneously two-dimensional maps of soot temperature and volume fraction in axisymmetric flames [12].

Two CW Lasers operating at $\lambda_{1}=645 \mathrm{~nm}$ (red) and $\lambda_{2}=785 \mathrm{~nm}$ (infrared) are used as the continuous light sources. The selection of these wavelengths results from a trade-off between the sensitivity of the temperature measurement and the signal-to-noise ratio. Furthermore, the use of the upper part of the visible spectrum consolidates the predominance of absorption over scattering by soot particles, knowing that the participating medium is considered as non-scattering in the post-processing. As a result, the selection of 
$\lambda_{1}$ and $\lambda_{2}$ was proven to enable the simultaneous reconstructions of the soot temperature and volume fraction distributions with acceptable uncertainties [12].

Each $1 \mathrm{~mm}$ diameter laser beam passes through a half-wave plate and a shutter that chops the source at a frequency tuned at $25 \mathrm{~Hz}$. A polarizing cube beamsplitter is used to transmit the red laser beam and reflect the infrared one. This arrangement allows the merged outcoming beam to be a combination of both wavelengths. Furthermore, the intensity of each beam can be tuned independently adjusting the angle between each half-wave plate and the polarizing cube. The subsequent optical arrangement then provides with a collimated beam with an outgoing diameter of $70 \mathrm{~mm}$. Across its section, the non-coherent light intensity is fairly uniform at both wavelengths.

After passing through the flame, the beam is decollimated. Upstream the focal point a beam splitter allows the reflection of $50 \%$ intensity and the transmission of the other $50 \%$ at both wavelengths. For each laser beam, a pinhole is located at the focal point. This provides a telecentric configuration possessing depth-invariant magnification and filters the slight beam steering due to the temperature gradient that could bias the deconvolution process. Every resulting virtual image formed is re-imaged by a 12-bit progressive scan monochrome camera mounted with a conventional lens and equipped with a narrow band filter. To enable the monochromatic imaging, these filters are centered at $645 \mathrm{~nm}$ and $785 \mathrm{~nm}$, and exhibit a band width at one half the transmissivity maximum of $20 \mathrm{~nm}$ and $10 \mathrm{~nm}$, respectively. Every CMOS camera array is composed of 1312x1082 pixels, providing a spatial resolution of $150 \mu \mathrm{m}$ for the projected data. 
A digital pulse generator controls the occurrence and the duration of every CMOS exposure, together with every shutter opening. A frame grabber records the frames captured by the cameras at a rate of 25 frames per second. In the present study, the exposure time needs to be adjusted for every oxygen content of the coflowing oxidizer as the level of intensity emitted by the flame is especially governed by this content. Consequently, the exposure time is set to $6.7,4.7$, and $2.7 \mathrm{~ms}$ for $\mathrm{X}_{\mathrm{O}_{2}, \mathrm{c}}=0.30,0.40$, and 0.50 , respectively.

The methodology of the deconvolutions that underly the MAE technique is briefly reminded here. It is extensively outlined in Ref.[12]. The flame is considered an emitting, absorbing, but non-scattering medium. For the laminar coflow partially premixed ethylene flames studied, the flame radiative spectrum in the visible is governed by the continuum radiation from soot. This is particularly true in the upper part of the visible spectrum. In addition, absorption by soot particles produced in these flames is shown to be at least one order of magnitude higher than scattering, especially at large wavelengths in the visible range [22]. In such a configuration, the Radiative Transfer Equation that models the transfer of the radiative intensity can be integrated along an optical pathway, such as the one followed by both collimated laser beams inside the flame in Fig.1.

When the laser is off, the energy $\mathcal{E}_{\lambda}^{\text {off }}$ accumulated on a pixel during a time $\Delta t$ due to the steady impinging flux emitted by the flame at wavelength $\lambda$. When the laser is on, the energy $\mathcal{E}_{\lambda}^{o n}$ accumulated on the same pixel is $\mathcal{E}_{\lambda}^{o f f}$ complemented by the energy deposited by the incident non-coherent light ray. Measuring consecutively $\mathcal{E}_{\lambda}^{(o n)}$ and $\mathcal{E}_{\lambda}^{(o f f)}$ allows the attenuation, i.e. the difference between both quantities, to be only connected to the spectral 
absorption coefficient field $\kappa_{\lambda}(r, z)$ crossed along the optical pathway leading to the pixel considered. The whole field $\kappa_{\lambda}(r, z)$ can then be retrieved by conducting the attenuation measurements for every pixel of the camera's sensor. The soot volume fraction field $f_{v}(r, z)$ can also be inferred. The Mie theory allows $\kappa_{\lambda}$ to be transformed into $f_{v}$, assuming that soot particles are in the Rayleigh limit [21]:

$$
f_{v}(r, z)=\left[\lambda \kappa_{\lambda}(r, z)\right] /[6 \pi E(m)]
$$

where $E(m)$ is a function of the complex refractive index $m$ of soot.

The reconstruction of the local spectral emission rate $\kappa_{\lambda} B_{\lambda}(r, z)$ emitted by the flame is now also allowed, $B_{\lambda}$ being the spectral blackbody radiative intensity at the local temperature $T$ given by the Planck's law:

$$
B_{\lambda}=\frac{2 h \pi c^{2}}{\lambda^{5}\left(e^{\frac{h c}{\lambda k T}}-1\right)}
$$

with $k=1.380710^{-23} \mathrm{~J} / \mathrm{K}$ being the Boltzmann constant, $h=6.62610^{-34} \mathrm{~J} . \mathrm{s}$ the Planck constant, and $c$ the speed of light in vacuum $\left(2.99810^{8} \mathrm{~m} / \mathrm{s}\right)$. The reconstruction of $\kappa_{\lambda} B_{\lambda}(r, z)$ consists of measuring $\mathcal{E}_{\lambda}^{o f f}$ for every pixel of the camera's sensor. $B_{\lambda}(r, z)$ can then be extracted when computing the ratio $\kappa_{\lambda} B_{\lambda} / \kappa_{\lambda}$. The map of soot temperature $T(r, z)$ can finally be inferred from a lookup table that provides $T$ as a funcion of the ratio $B_{\lambda_{2}} / B_{\lambda_{1}}$. This table accounts for the different characteristics of the CMOS sensors and the overall spectral efficiencies along the collection optical pathways as a correcting factor is determined using a Halogen calibration light source that is imaged on both CMOS sensors.

In practice, at every height $z_{i}$ imaged on a line of pixels, every aforementioned deconvolution leads to a system of linear equations that is solved for 
$\left(\kappa_{\lambda}\right)_{i j}$ and $\left(\kappa_{\lambda} B_{\lambda}\right)_{i j}$, respectively, at the locations $r_{j}$ along the line. As every set of equations is shown to be ill-conditioned, a Tikhonov regularization is used to stabilize the deconvolution process [12].

In the following, $f_{v}(r, z)$ is inferred from $\kappa_{\lambda_{2}}(r, z)$ since for a given fuel $E(m)$ tends towards a constant value when $\lambda$ increases within the near infrared spectrum [16]. In addition, using the higher wavelength further consolidates the validity of the aforementioned Rayleigh limit. As proceeded in former studies [21, 8], $E(m)$ is here adjusted to 0.43 to reproduce the peak soot volume fraction measured by Santoro et al. [17] at $\mathrm{HAB}=50 \mathrm{~mm}$ for ethylene burning in air.

Furthermore, following the methodology prescribed by Legros et al. [12], confidence regions for soot temperature and volume fraction measurements can be identified according to the local relative levels of $\kappa_{\lambda} B_{\lambda}$ and $\kappa_{\lambda}$. These regions are also identified here, guaranteeing that the measurements of soot temperature and volume fraction exhibit uncertainty levels lower than $\pm 50 \mathrm{~K}$ and $\pm 0.5 \mathrm{ppm}$, respectively. Still, the latter does not incorporate the uncertainty associated with $E(m)$ due to the ongoing debate about this quantity mentioned in the introduction.

Finally, the fields of soot temperature and volume fraction shown in the following result from the deconvolution of the signals captured on the right hand side of the flame's axis. The fields inferred from the left hand side of the flame lead to very similar results that do not influence the ultimate findings about the effects of the magnetic gradient on soot production in the flames investigated. 


\section{Results and discussion}

\subsection{Soot temperature and volume fraction fields}

Figure 2 shows the fields of non-dimensional soot volume fraction and temperature, for increasing magnetic gradient from the left to the right, for decreasing equivalence ratio from the top to the bottom, and for $\mathrm{X}_{\mathrm{O}_{2}, \mathrm{c}}=0.3$. The maximum value $f_{v, \max }$ of soot volume fraction can be considered a relevant characteristic of the competition between soot formation and oxidation in a given flame. For this reason, the height $z_{\max }$ at which $f_{v, \max }$ occurs is also a characteristic length of the soot formation and oxidation processes. Thus, for every equivalence ratio, a characteristic couple $\left(z_{\max }, f_{v, \max }\right)$ is defined in the absence of magnetic gradient (MagF 0). These are specified for every line in Fig. 2 that exhibits the field of non-dimensional $f_{v} / f_{v, \max }$ and its evolution as a function of the non-dimensional coordinates $z / z_{\max }$ and $r / d_{F}$. Similarly, $(T-300) /\left(T_{\max }-300\right)$ is shown. $T_{\max }$ is the maximum soot temperature measured for MagF 0 and the considered equivalence ratio.

For the non-premixed flames $(\phi=\infty)$, the tendencies reported by Jocher et al. [8] are reproduced. With increased magnetic gradient, the soot layer is broadened. However, this trend is balanced as the peak soot volume fraction slightly decreases in magnitude from MagF 2 to MagF 3. To assess the budget of these opposing effects, net soot production can be characterized by the mean integrated soot volume fraction $F_{v}(z)$ defined as follows:

$$
F_{v}(z)=\frac{4}{\pi d_{F}^{2}} \int_{0}^{d_{F} / 2} 2 \pi r f_{v}(r, z) d r
$$

$F_{v}(z)$ appears as an evaluation of the actual soot load at a given HAB in the flame. Figure 3 exhibits the profiles of $F_{v}$ as a function of non-dimensional 
height $z / z_{\max }$, for every magnetic gradient investigated, for three different values of the equivalence ratio, and for $\mathrm{X}_{\mathrm{O}_{2}, \mathrm{c}}=0.3$ and 0.4 . Thus, at $\phi=\infty$ and for both $\mathrm{X}_{\mathrm{O}_{2}, \mathrm{c}}$ levels, increasing the magnetic gradient monotonically enhances soot production. Furthermore, when increasing $\mathrm{X}_{\mathrm{O}_{2}, \mathrm{c}}$, the enhancement of the soot production by the magnetic gradient is more pronounced.

As argued by Jocher et al. [8], the paramagnetic oxygen molecules are attracted upwards due to the magnetic force per unit mass $\chi_{O_{2}} \nabla\left(\mathbf{B}^{2}\right) /\left(2 \mu_{0}\right)$ experienced along the streamlines from the burner surface towards the horizontal axis of symmetry of the iron coils [23]. $\mu_{0}$ and $\chi_{\mathrm{O}_{2}}$ are here the vacuum magnetic permeability and the positive oxygen magnetic susceptibility per unit mass, respectively. As the magnitude of $\chi_{\mathrm{O}_{2}}$ can be assumed several times larger than those of diamagnetic gases [24], the main influence of the magnetic field on the flame is expected to be caused by the oxygen concentration field. As shown for this configuration by Yamada et al. [24] and Wakayama et al. [25], this results in a thermo-magneto convection. Indeed, the buoyant force per unit volume $\mathbf{f}_{b}$ that is directed upwards in a flame can be expressed as:

$$
\mathbf{f}_{b}=\left(\rho-\rho^{(\infty)}\right) \mathbf{g},
$$

where $\mathbf{g}$ is the gravitational acceleration and $\rho$ the local fluid density. The superscript $(\infty)$ indicates here the conditions away from the flame. Manipulating the formulation of the aforementioned magnetic force per unit mass in a similar manner leads to the following expression of the magnetic force per unit volume $\mathbf{f}_{\text {mag }}$ that initiates and sustains the thermo-magneto convection:

$$
\mathbf{f}_{m a g}=\left(\rho Y_{O_{2}} \chi_{O_{2}}-\rho^{(\infty)} Y_{O_{2}}^{(\infty)} \chi_{O_{2}}^{(\infty)}\right) \frac{\nabla\left(\mathbf{B}^{2}\right)}{2 \mu_{0}}
$$

with $Y_{\mathrm{O}_{2}}$ being the oxygen mass fraction. 
Following Eq.(5), an upward $\nabla\left(\mathbf{B}^{2}\right)$ can generate a force that is opposed to buoyancy. As a result, the global residence time inside the flame is increased when increasing the magnitude of the upward $\nabla\left(\mathbf{B}^{2}\right)$. This effect that can be considered similar to a decrease in $\mathbf{g}$ magnitude then tends to locally promote soot formation [26]. In addition, the magnetic force will also reduce the velocity of the fresh oxidizer flow. The shear stress between the oxidizer and fuel flow will therefore be weakened. This further enhances the magnetic influence on the soot formation process.

On the contrary, the magnetic effect on the soot oxidation process is more ambiguous. Within the region where soot oxidation starts prevailing, i.e. for $z / z_{\max } \geq 1$, the increased residence time tends to also boost the oxidation process. Concomitantly, radiative heat losses from the soot particles are increased with increasing magnetic gradient due to the larger amount of soot produced downstream $z / z_{\max }=1$. The latter influence seems to dominate as evidenced by the evolution of the temperature fields for $\phi=\infty$ in Fig. 2. Indeed, the temperature in the vicinity of the flame's axis is decreased when the magnetic gradient is increased. This is in agreement with the aforementioned spread of the soot layer towards the flame's axis where soot oxidation is delayed due to this decrease in temperature.

When increasing the equivalence ratio for MagF 0, the peak soot volume fraction first remains on the edge of the flame for $\phi=6.8$, like in the nonpremixed flames. However, the soot production is significantly enhanced in the vicinity of the flame's axis, making the soot volume fraction field more uniform. For $\phi=5$, the wing of this field has almost been smoothed. Indeed, due to the premixed feature of these flames, the inner temperature 
in the vicinity of the axis is higher than that of the non-premixed flame. In addition, for rich premixed flames ( $\phi=6.8$ and 5 ), the fuel that has not been oxidized within the primary reaction zone remains in a significant amount downstream, which leads to higher soot formation rates within the central part of the flame as compared to the non-premixed configuration. These trends are in qualitative agreement with the experimental and numerical profiles of soot volume fraction delivered by Arana et al. [10] and Chernov et al. [11]. Similarly, the soot temperature field exhibits a more uniform front width along the flame height as $\phi$ is increased.

The effect of the magnetic gradient evidenced for the non-premixed flames is first corroborated by the fields measured for $\phi=6.8$ and MagF 1 . The soot layer also spreads over towards the flame's axis. However, due to the more uniform temperature front in this premixed flame, radiative heat losses then affect the temperature front, therefore the soot oxidation rate to a relatively uniform extent. As a result of the enhanced soot formation, the complete soot oxidation is delayed and the flame is longer. Further increasing the magnetic gradient to MagF 2, the soot formation starts decreasing. Finally, for MagF 3, the initial trend has definitely reversed and the inner soot layer collapses. The wing of the soot layer that cannot be displayed on this map then looks more distinct. These observations are confirmed by the evolutions of $F_{v}$ in Fig.3. For $\phi=6.8$ and $\mathrm{X}_{\mathrm{O}_{2}, \mathrm{c}}=0.3$, the soot production is first increased with increasing magnetic gradient but then decreased from MagF 1 to MagF 3 . Interestingly, this reversal occurs at a higher magnetic gradient, i.e. MagF 2 , for $\mathrm{X}_{\mathrm{O}_{2}, \mathrm{c}}=0.4$.

For $\phi=5$, the fields displayed in Fig.2 and the profiles shown in Fig.3 
demonstrate that the initial effect of the magnetic gradient emerges again. Indeed, increasing the magnetic gradient leads to a monotonic intensification of soot formation though the magnitude of the overall effect has been reduced. Locally, a restricted region in the vicinity of the axis exhibits a slight decrease in soot volume fraction.

For brevity, data extracted from the flames established for $\mathrm{X}_{\mathrm{O}_{2}, \mathrm{c}}=0.5$ are not shown. No reversal of the magnetic effect could be observed for these conditions.

\subsection{Parameters governing the magnetic effects}

According to Curie's law, the oxygen magnetic susceptibility scales with $1 / T[24]:$

$$
\chi_{O_{2}}=\frac{N_{A} g_{L}^{2} \mu_{B}^{2} S_{O_{2}}\left(S_{O_{2}}+1\right) \mu_{0}}{3 k m_{O_{2}}} \frac{1}{T}
$$

where $N_{A}$ is the Avogadro number, $g_{L}$ the Lande's g-factor, $\mu_{B}$ the Bohr magneton, $S_{\mathrm{O}_{2}}$ the total angular momentum of electron spin, $k$ the Boltzmann constant, and $m_{\mathrm{O}_{2}}$ the oxygen molar weight. Calling $C$ the constant equal to $\left(N_{A} g_{L}^{2} \mu_{B}^{2} S_{O_{2}}\left(S_{O_{2}}+1\right)\right) /\left(6 k m_{O_{2}}\right)$, Eq.(5) can be combined with the ideal gas law and formulated as follows:

$$
\mathbf{f}_{m a g}=C\left(\frac{1}{T} \frac{X_{O_{2}} / T-X_{O_{2}}^{(\infty)} / T^{(\infty)}}{X_{O_{2}}^{(\infty)} / T^{(\infty)}}-\frac{1}{T} \frac{T-T^{(\infty)}}{T^{(\infty)}}\right) \rho^{(\infty)} Y_{O_{2}}^{(\infty)} \nabla\left(\mathbf{B}^{2}\right)
$$

Eq.(7) highlights the thermo-chemical coupling that the magnetic force sustains in the momemtum equation. Eq.(7) also shows that the effect of the magnetic gradient can be potentially reversed according to the sign of the overall term between brackets, i.e. in some part of the flame $1 / T\left(X_{\mathrm{O}_{2}} / T-\right.$ $\left.X_{O_{2}}^{(\infty)} / T^{(\infty)}\right) /\left(X_{O_{2}}^{(\infty)} / T^{(\infty)}\right)$, hereafter referred to as the thermo-chemical term, 
can balanced the term $1 / T\left(T-T^{(\infty)}\right) / T^{(\infty)}$, referred to as the thermal term in the following.

Figure 4(a1) displays the schematic profiles of non-dimensional temperature and oxygen mole fraction in a non-premixed flame. At the arbitrary location $r_{n p f} / d_{F}=0.3, T_{\max }$ has been set here to $2077 \mathrm{~K}$, which is the peak soot temperature measured for $\phi=\infty$ and $\mathrm{X}_{\mathrm{O}_{2}, \mathrm{c}}=0.3$. Although equating the maximum flame temperature with that of soot sounds questionable, the level of $T_{\max }$ hardly affects the different kinds of regions that are identified in the following. As shown in Fig.4(b1), due to the absence of oxygen on the fuel side of the flame, the thermo-chemical term tends towards $-1 / T$ on this side. As the thermal term is always negative, the whole flame will be systematically submitted to a thermo-magneto convection that points downwards, therefore is opposed to buoyancy. Most of the soot is located on the flame's wing (see the upper row in Fig.2) and radiative heat loss by soot reduces the flame temperature. Thus, the overall term still induces a relatively high downward magnetic force that leads to a significant increase in the residence time, therefore promotes soot formation. This statement further supports the qualitative reasoning by Jocher et al. [8].

Figure 4(a2) displays the schematic profiles of temperature and oxygen mole fraction in a partially premixed flame at HAB close to the burner nozzle. In this specific configuration, $\mathrm{X}_{\mathrm{O}_{2}}$ is potentially relatively high in the fuel mixture in the vicinity of the flame's axis. Moving away from the axis, all the oxygen is first consumed in the rich premixed flame, arbitrarily located here around $r_{p f} / d_{F}=0.2$. As a result of the moderate heat release, the temperature rises to an intermediate level. At $r_{n p f} / d_{F}=0.3$, the remaing fuel 
is burnt in the diffusion flame, where the temperature reaches $T_{\max }$. Finally $\mathrm{X}_{\mathrm{O}_{2}}$ and $T$ tend towards the conditions met away from the flame in the oxidizer stream. To compute the thermo-chemical and thermal terms shown in Fig.4(b2), two flames, called A and B, are considered. Flame A corresponds to $\phi=6.8$ and $X_{O_{2}, c}=0.3$, for which $X_{O_{2}, \max }$ is 0.31 and the measured $T_{\max }$ is $2242 \mathrm{~K}$. Flame $\mathrm{B}$ corresponds to $\phi=6.8$ and $X_{O_{2}, c}=0.5$, for which $X_{O_{2}, \max }$ is 0.5 and the measured $T_{\max }$ is $2584 \mathrm{~K}$. The major discrepancy between flames A and B is the sign of the thermo-chemical term. For flame A, this term is positive and high enough to control the sign of the overall term. On the contrary, for flame B, the thermo-chemical term is slightly negative and the sign of the overall term is controlled by the thermal term. Thus, the inner region of Flame A experiences a thermo-magneto convection pointing upwards, which contributes to a shorter residence time, therefore the mitigation of soot formation. On the contrary, Flame B is not affected by such a reversed effect.

These findings are in agreement with the aforementioned experimental observations, i.e the shrinking of the inner soot layer at elevated magnetic gradient for $\phi=6.8$ and $X_{O_{2}, c}=0.3$ (see the mid row in Fig.2) and the higher magnetic gradient required to reverse the magnetic effect when increasing $X_{O_{2}, c}$ (see the profiles for $\phi=6.8$ in Fig.3). Furthermore, increasing the equivalence ratio at a given $X_{O_{2}, c}$ leads to a shift of the premixed flame location $r_{p f} / d_{F}$ towards the centerline together with a higher inner temperature, as shown along the first column in Fig.2. Consequently, the region of influence of the upward magnetic force is very restricted, which is consistent with the absence of magnetic effect reversal for $\phi=5$. 


\section{Conclusion}

The present study may be considered a further step towards the identification of a possible strategy that would enable the magnetic control of soot production in partially premixed reacting flows. The original experiments were conducted with the Santoro coflow burner together with an optical arrangement that allows the MAE technique to be implemented. As a result, fields of both soot temperature and volume fraction were used to assess the influence of an upward magnetic gradient on the soot production inside laminar partially premixed ethylene/oxygen flames. A reversal of the magnetic effect was revealed. On one hand, for non-premixed flames, increasing the upward magnetic gradient systematically led to an increase in soot production. This was attributed to an enhanced residence time due to the increased downward magnetic force. On the other hand, for some partially premixed flames, the magnetic gradient could induce an overall reduction in soot production. Theoretical profiles of temperature and oxygen mole fraction in the flame were scaled by the measurements obtained and helped identify the relative importance of a thermo-chemical and a thermal contributions to the magnetic force. In the inner part of some partially premixed flames, the thermo-chemical contribution may control the reversal of the effect as the magnetic force then points locally upwards. This leads to a local decrease of the residence time, therefore a reduction of soot production.

The original identification of these dual magnetic effects now supports the need for subsequent numerical simulations. These will especially allow for the computation of the actual momentum budget in these partially premixed flames experiencing magnetic gradients. The optimization of one or both 
of the effects requires the evaluation of their relative local magnitude with respect to other potentially governing forces, such as buoyancy.

As an illustration of a potential magnetic control of soot release, future efforts will be devoted to the identification of the possible magnetic effect on the inhibition of sooting flame flickering. Indeed, the onset of this instability is very sensitive to the amount of soot produced in the flame. Such an extended domain of combustion completeness was supposed to be the source of significant fuel savings when permanent magnets were located around the fuel injector in an internal combustion engine [27]. However, no experimental evidence has been shown on an academic configuration up to now. 


\section{References}

[1] M. Patricia Sierra-Vargas, L.M. Teran, Respirology 17 (2012) 1031-1038.

[2] P.T. O' Shaughnessy, Environ. Sci.: Processes Impacts 15 (2013) 49-62.

[3] P. Tandon, A. Heibel, J. Whitmore, N. Kekre, K. Chithapragada, Chem. Eng. Sci. 65 (2010) 4751-4760.

[4] K. Leistner, A. Nicolle, P. Da Costa, Energy \& Fuels 26 (2012) 6091-6097.

[5] R.J.H. Klein-Douwel, A.J. Donkerbroek, A.P. van Vliet, M.D. Boot, L.M.T. Somers, R.S.G. Baert, N.J. Dam, J.J. ter Meulen, Proc. Combust. Inst. 32 (2009) 2817-2825.

[6] O. Angrill, H. Geitlinger, T. Streibel, R. Suntz, H. Bockhorn, Proc. Combust. Inst. 28 (2000) 2643-2649.

[7] Y. Wang, G.J. Nathan, Z.T. Alwahabi, K.D. King, K. Ho, Q. Yao, Comb. Flame 157 (2010) 1308-1315.

[8] A. Jocher, H. Pitsch, T. Gomez, G. Legros, Proc. Combust. Inst. 35 (2015) 889-895.

[9] C.S. McEnally, L.D. Pfefferle, Comb. Flame 121 (2000) 575-592.

[10] C.P. Arana, M. Pontoni, S. Sen, I.K. Puri, Comb. Flame 138 (2004) 362-372.

[11] V. Chernov, Q. Zhang, M.J. Thomson, S.B. Dworkin, Comb. Flame 159 (2012) $2789-2798$.

[12] G. Legros, Q. Wang, J. Bonnety, M. Kashif , C. Morin, J.-L. Consalvi, F. Liu, Combust. Flame 162 (2015) 2705-2719.

[13] F. Liu, K.A. Thomson, G.J. Smallwood, Combust. Flame 160 (2013) 1693-1705.

[14] H. Zhao, B. Williams, R. Stone, J. Quant. Spectrosc. Radiat. Transf. 133 (2014) $136-152$.

[15] F. Goulay, P.E. Schrader, H.A. Michelsen, Appl Phys B 100 (2010) 655-663.

[16] J. Yon, R. Lemaire, E. Therssen, P. Desgroux, A. Coppalle, K.F. Ren, Appl Phys B 104 (2011) 253-271.

[17] R.J. Santoro, H.G. Semerjian, R.A. Dobbins, Combust. Flame 51 (1983) 203-218.

[18] F. Liu, G. Hongsheng, G.J. Smallwood, Ö.L. Gülder, Combust. Theory Modelling 7 (2003) 301-315.

[19] C.S. McEnally, L.D. Pfefferle, Environ. Sci. Technol. 45 (2011) 2498-2503.

[20] G. Legros, T. Gomez, M. Fessard, T. Gouache, T. Ader, P. Guibert, P. Sagaut, J.L. Torero, Proc. Combust. Inst. 33 (2011) 1095-1103. 
[21] M. Kashif, J. Bonnety, P. Guibert, C. Morin, G. Legros, Opt. Express 20 (2012) $28742-28751$.

[22] F. Liu, D.R. Snelling, K.A. Thomson, G.J. Smallwood, Appl Phys B 96 (2009) 623636.

[23] N. Wakayama, Chemical Physics Letters 185 (1991) 449-451.

[24] E. Yamada, M. Shinoda, H. Yamashita, K. Kitagawa, Comb. Flame 135 (2003) 365379 .

[25] N. Wakayama, H. Ito, Y. Kuroda, O. Fujita, K. Ito, Comb. Flame 107 (1996) 187-192.

[26] G. Legros, J.L. Torero, Proc. Combust. Inst. 35 (2015) 2545-2553.

[27] R.J. Twardzik, Apparatus for subjecting hydrocarbon-based fuels to intensified magnetic fields for increasing fuel burning efficiency, US Patent 5,558,765 (1996). 


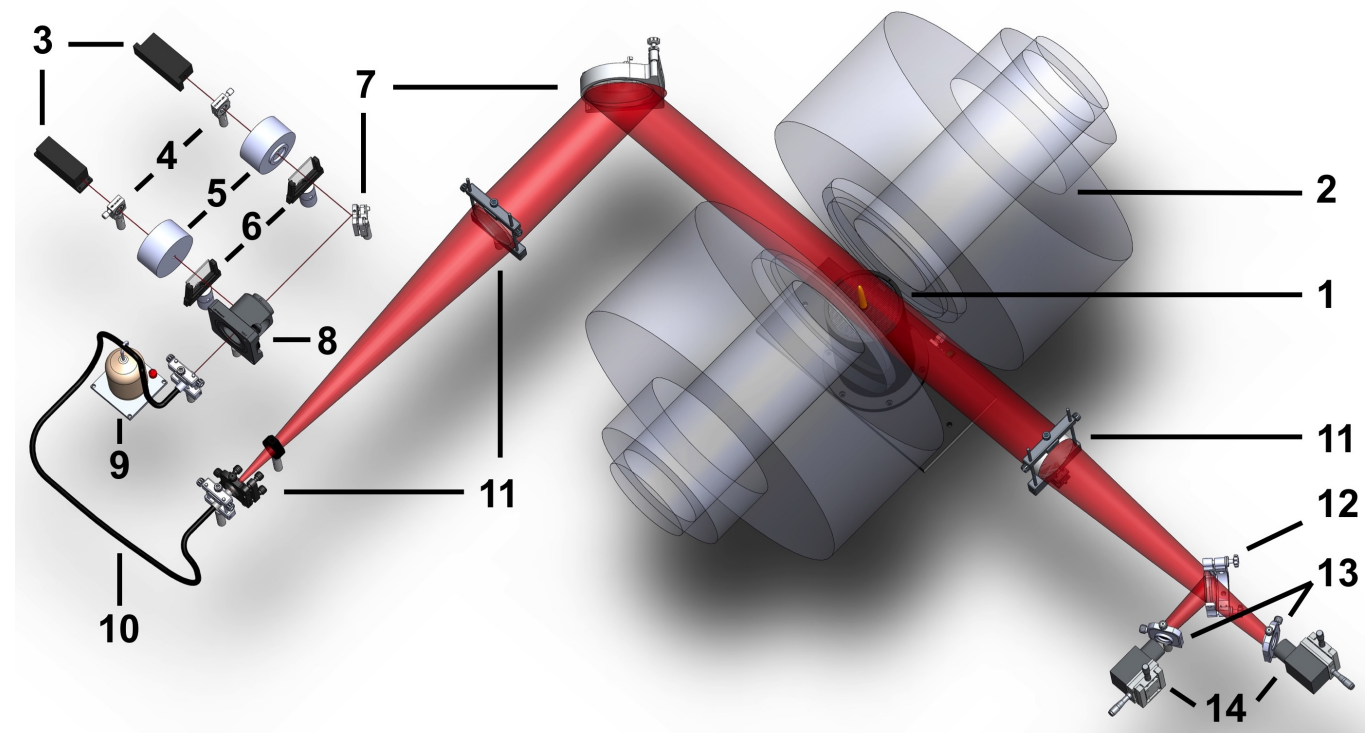

Figure 1: Schematic of the arrangement allowing partially premixed flames to be probed by the two-dimensional Modulated Absorption/Emission technique while exposed to different levels of $\nabla\left(\mathbf{B}^{2}\right)$. (1) Burner; (2) coils; (3) CW lasers; (4) half-wave plates; (5) shutters; (6) neutral density filters; (7) mirrors; (8) polarizer cube; (9) vibrational element; (10) optical fibre; (11) achromatic lenses; (12) beam splitter; (13) pinholes \& narrow band filters; (14) cameras. 


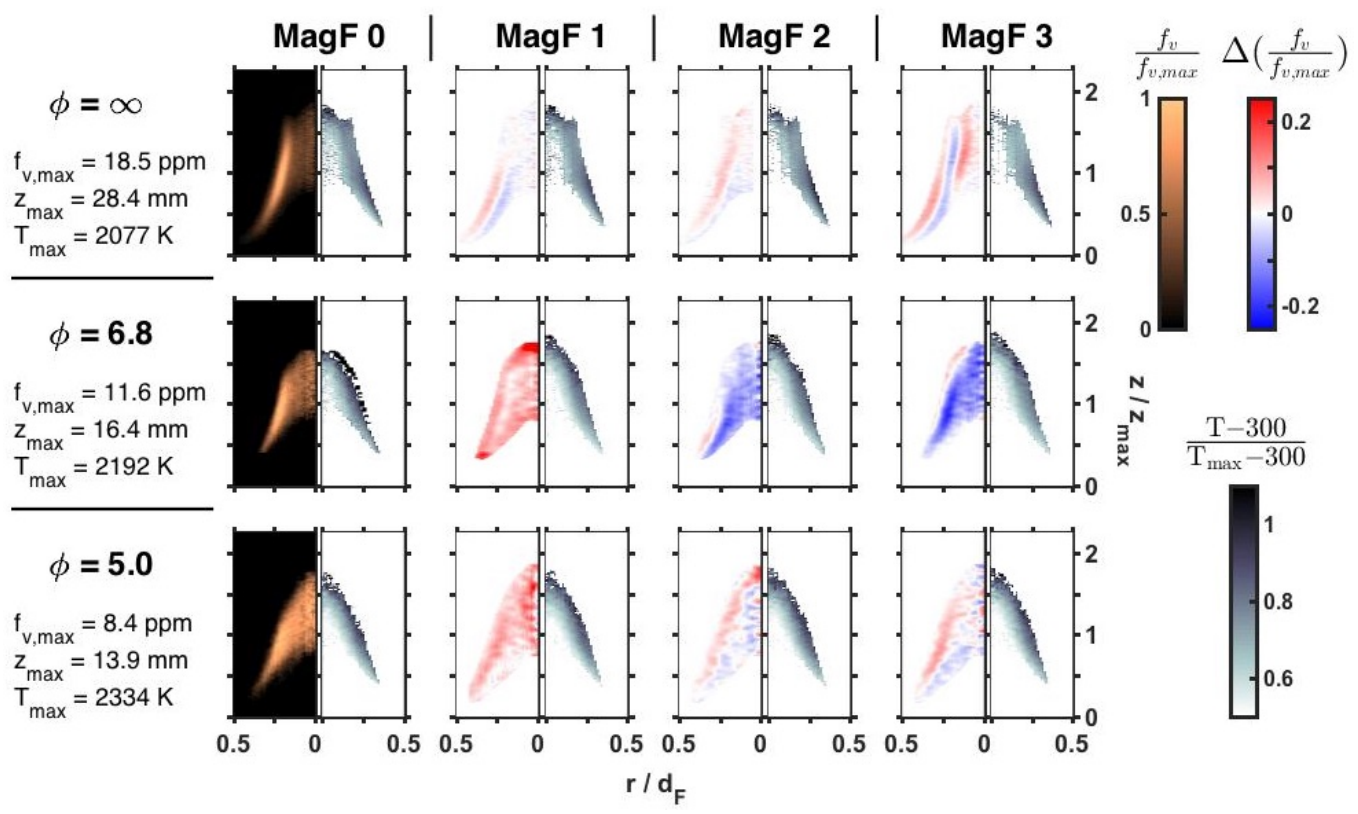

Figure 2: Fields of non-dimensional soot volume fraction and soot temperature for four levels of magnetic gradient, three different values of $\phi$, and $X_{0_{2}, c}=0.3$. For every value of $\phi$, the reference values $\left(z_{\max }, f_{v, \max }, T_{\max }\right)$ measured for MagF 0 are indicated on the left. From MagF 1 to MagF 3, the evolution of the soot volume fraction field is displayed. $\Delta\left(f_{v} / f_{v, \max }\right)$ is the difference between the non-dimensional soot volume fraction measured for the considered magnetic gradient and the one measured for the nearest lower magnetic gradient. 


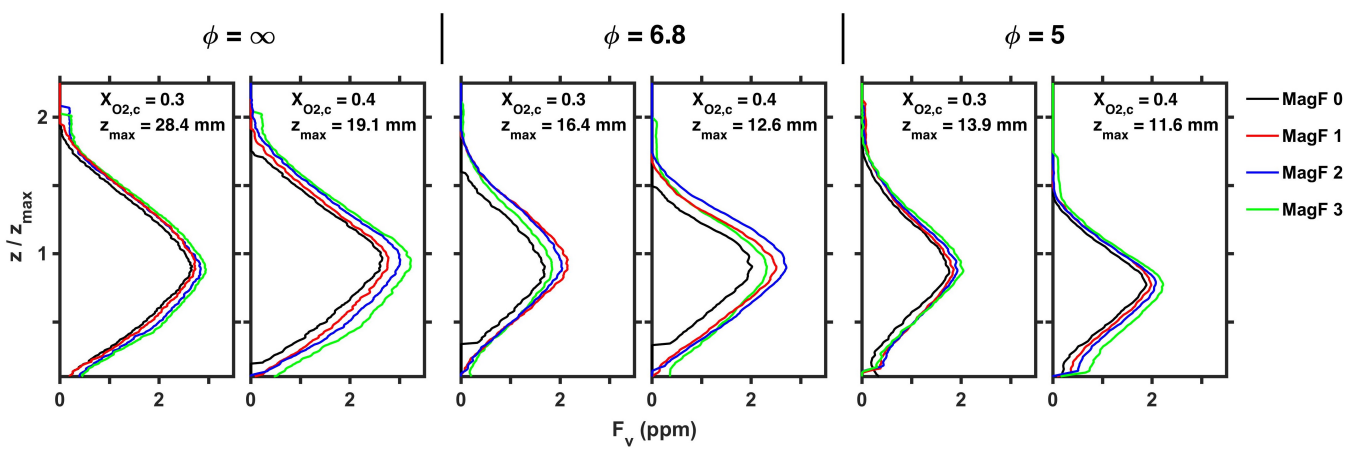

Figure 3: Profiles of net soot production $F_{v}$ along non-dimensional HAB for two levels of $X_{\mathrm{O}_{2}, c}$, for three different values of $\phi$, and for all the magnitudes of magnetic gradient investigated. 

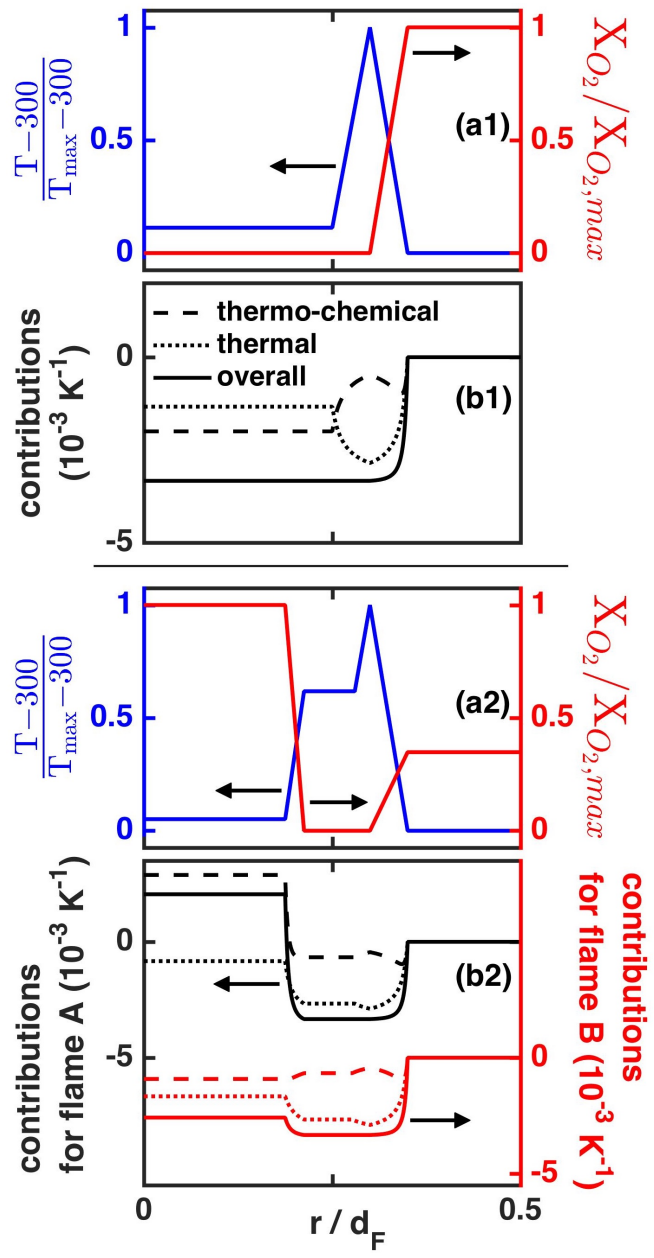

Figure 4: (a1) and (a2): schematic profiles of non-dimensional temperature and oxygen mole fraction in non-premixed and partially premixed flames, respectively; (b1) and (b2): evolution of the different contributions to the overall term in brackets in Eq.(7) for a nonpremixed flame and two partially premixed flames, respectively. For the non-premixed flame $\left(\phi=\infty\right.$ and $\left.X_{O_{2}, c}=0.3\right), T_{\max }=2077 \mathrm{~K}$ and $X_{O_{2}, \max }=0.3$. For flame A ( $\phi=6.8$ and $\left.X_{O_{2}, c}=0.3\right), T_{\max }=2242 \mathrm{~K}$ and $X_{O_{2}, \max }=0.31$. For flame $\mathrm{B}\left(\phi=6.8\right.$ and $\left.X_{O_{2}, c}=0.5\right)$, $T_{\max }=2584 \mathrm{~K}$ and $X_{O_{2}, \max }=0.5$. 


\section{List of Figures}

1 Schematic of the arrangement allowing partially premixed flames to be probed by the two-dimensional Modulated Absorption/Emission technique while exposed to different levels of $\nabla\left(\mathbf{B}^{2}\right)$. (1) Burner; (2) coils; (3) CW lasers; (4) half-wave plates; (5) shutters; (6) neutral density filters; (7) mirrors; (8) polarizer cube; (9) vibrational element; (10) optical fibre; (11) achromatic lenses; (12) beam splitter; (13) pinholes \& narrow band filters; (14) cameras. . . . . . . . . .

2 Fields of non-dimensional soot volume fraction and soot temperature for four levels of magnetic gradient, three different values of $\phi$, and $X_{0_{2}, c}=0.3$. For every value of $\phi$, the reference values $\left(z_{\max }, f_{v, \max }, T_{\max }\right)$ measured for MagF 0 are indicated on the left. From MagF 1 to MagF 3, the evolution of the soot volume fraction field is displayed. $\Delta\left(f_{v} / f_{v, \max }\right)$ is the difference between the non-dimensional soot volume fraction measured for the considered magnetic gradient and the one measured for the nearest lower magnetic gradient. . . . . . . . . . . . . . . . . .

3 Profiles of net soot production $F_{v}$ along non-dimensional HAB for two levels of $X_{\mathrm{O}_{2}, c}$, for three different values of $\phi$, and for all the magnitudes of magnetic gradient investigated. . . . . . . . . .

4 (a1) and (a2): schematic profiles of non-dimensional temperature and oxygen mole fraction in non-premixed and partially premixed flames, respectively; (b1) and (b2): evolution of the different contributions to the overall term in brackets in Eq.(??) for a nonpremixed flame and two partially premixed flames, respectively. For the non-premixed flame $\left(\phi=\infty\right.$ and $\left.X_{O_{2}, c}=0.3\right), T_{\max }=2077 \mathrm{~K}$ and $X_{O_{2}, \max }=0.3$. For flame $\mathrm{A}\left(\phi=6.8\right.$ and $\left.X_{O_{2}, c}=0.3\right), T_{\max }=2242 \mathrm{~K}$ and $X_{O_{2}, \max }=0.31$. For flame $\mathrm{B}\left(\phi=6.8\right.$ and $\left.X_{O_{2}, c}=0.5\right), T_{\max }=$ $2584 \mathrm{~K}$ and $X_{O_{2}, \max }=0.5 \ldots \ldots \ldots \ldots$ 


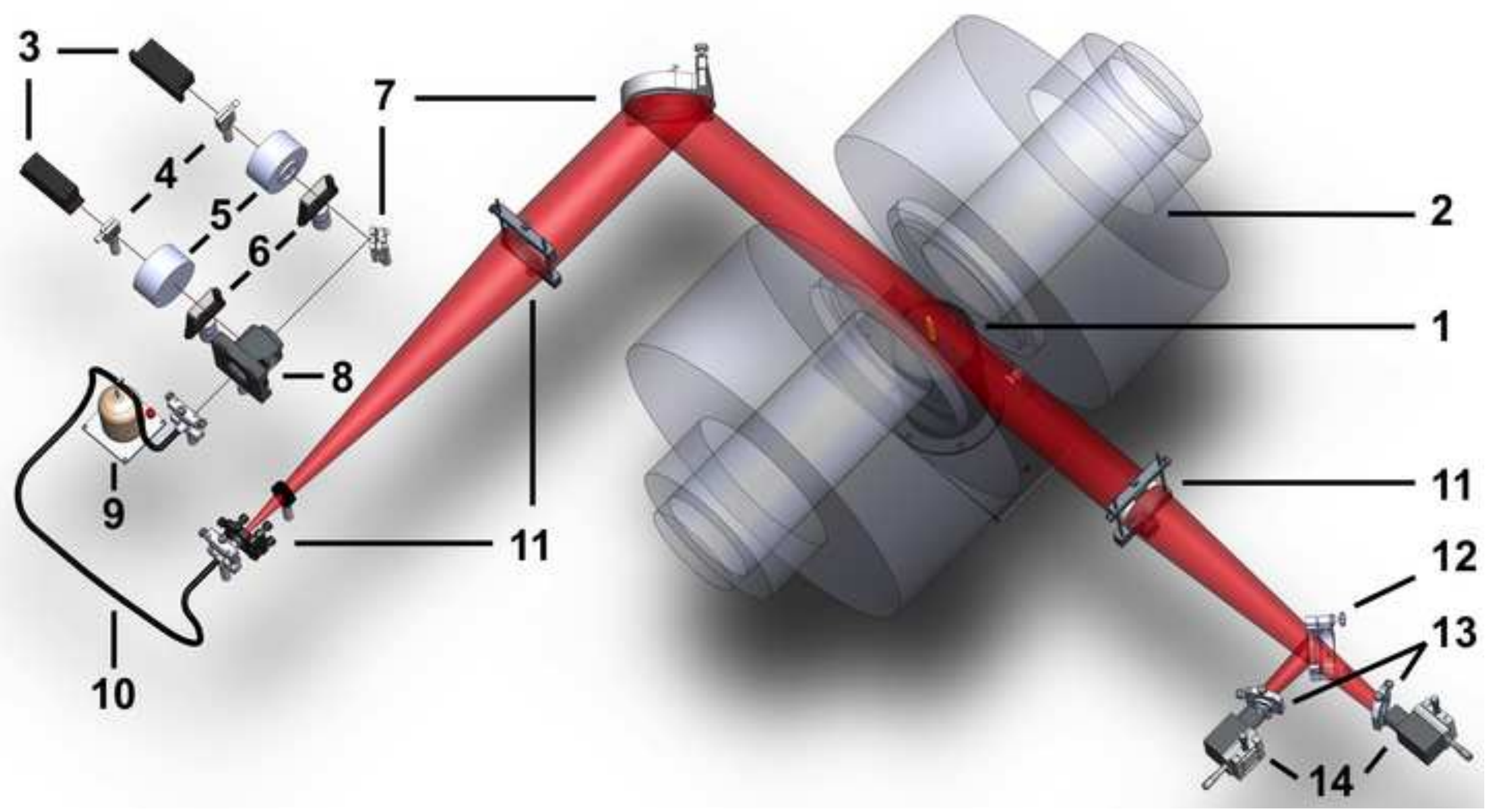




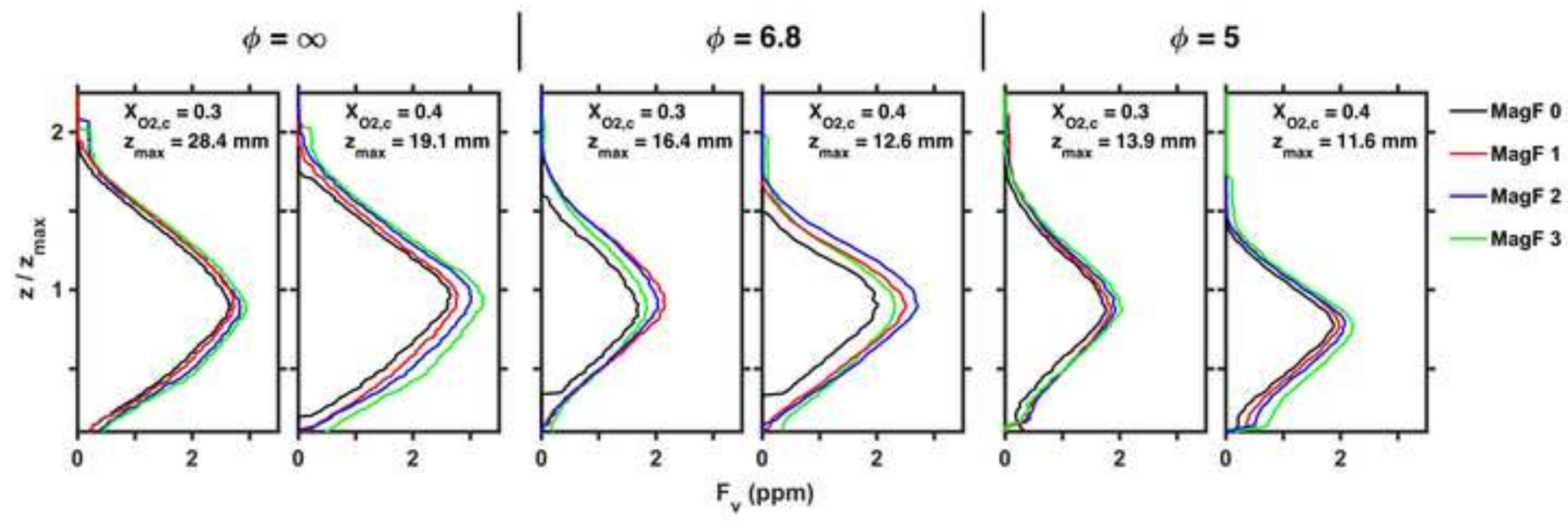



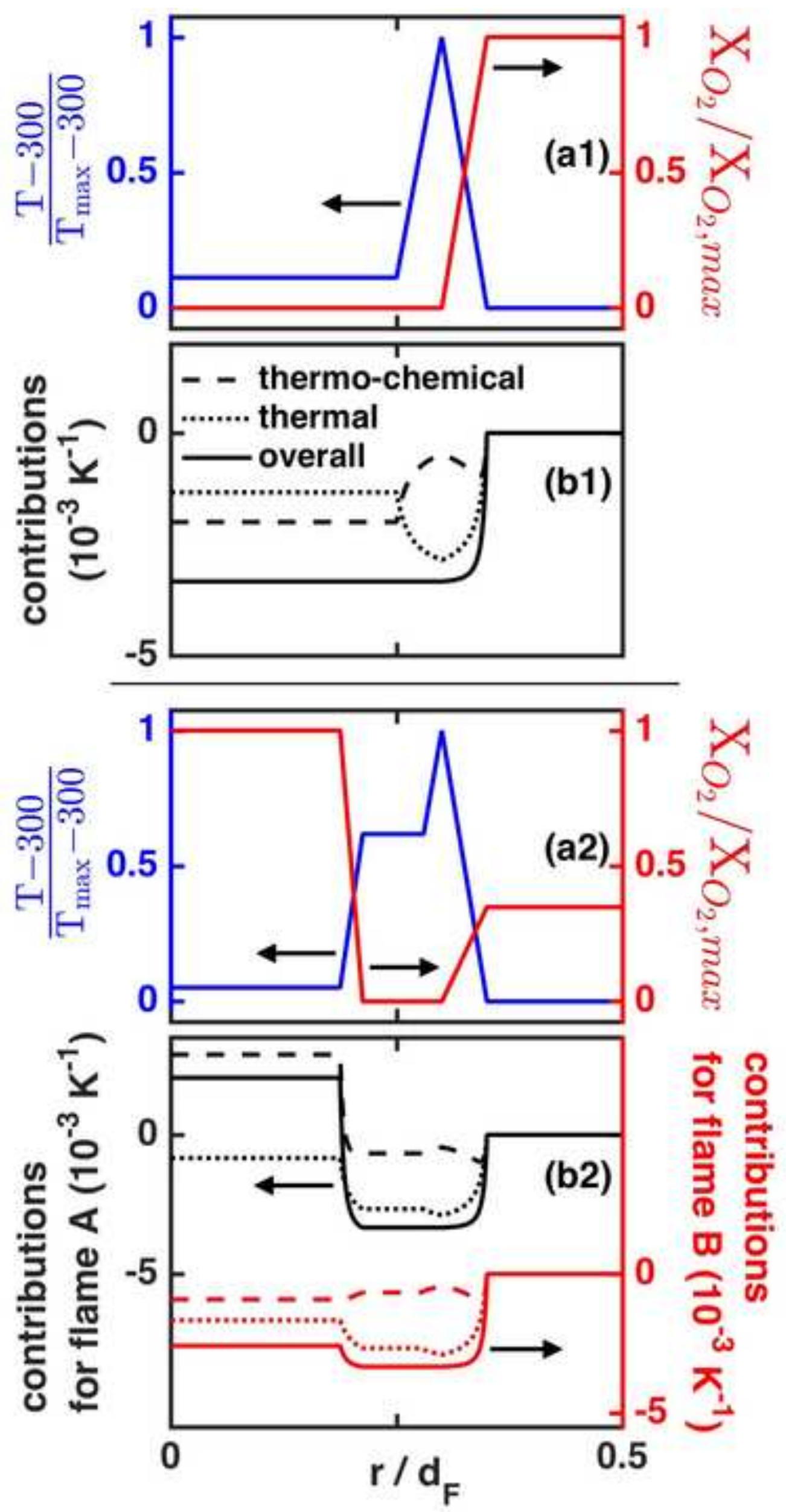


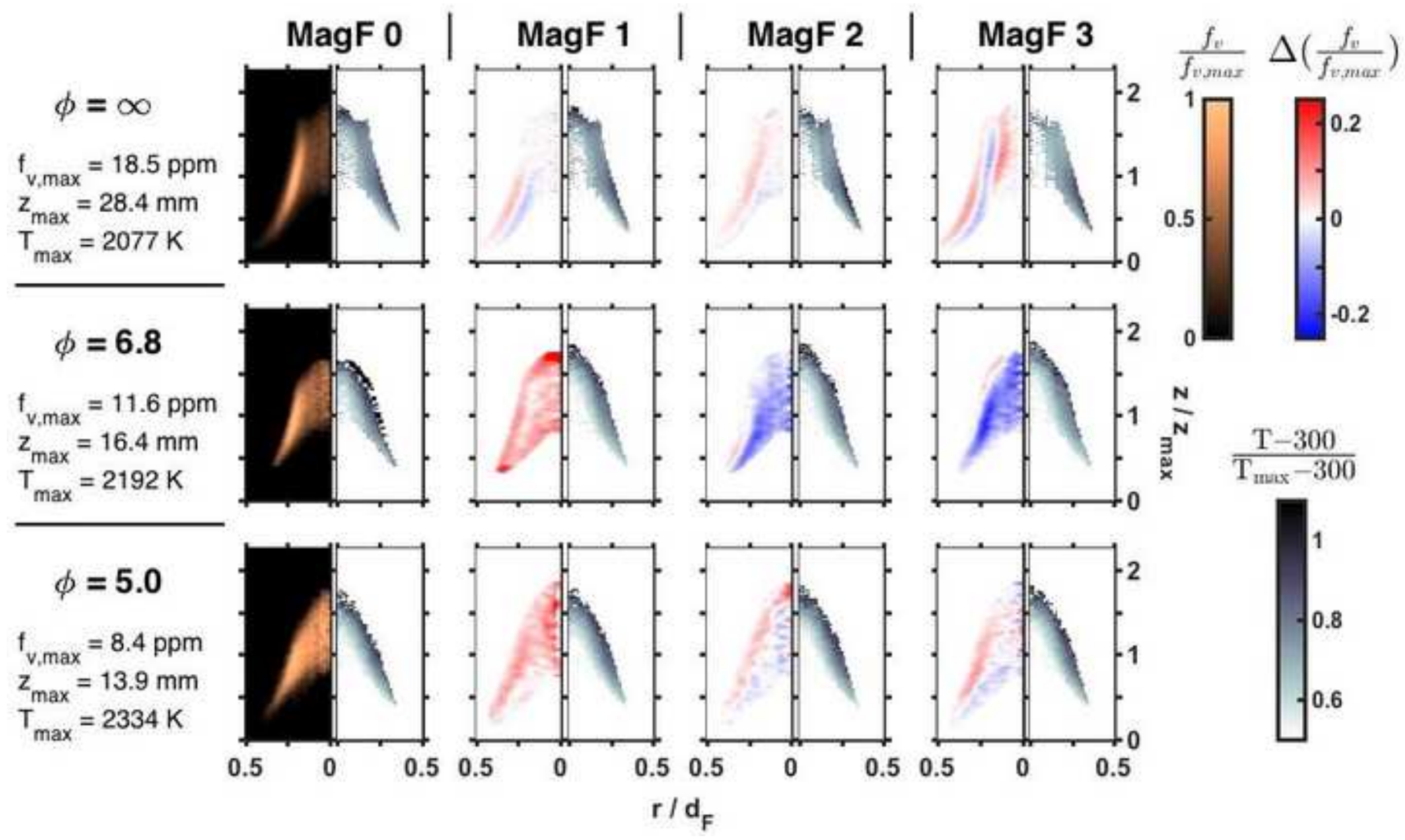

$$
\begin{aligned}
\phi & =\infty \\
\mathrm{f}_{\mathrm{v}, \text { max }} & =18.5 \mathrm{ppm} \\
\mathrm{z}_{\text {max }} & =28.4 \mathrm{~mm} \\
\mathrm{~T}_{\text {max }} & =2077 \mathrm{~K} \\
\phi & =6.8 \\
\mathrm{f}_{\mathrm{v}, \text { max }} & =11.6 \mathrm{ppm} \\
\mathrm{z}_{\text {max }} & =16.4 \mathrm{~mm} \\
\mathrm{~T}_{\text {max }} & =2192 \mathrm{~K} \\
\hline \phi & =5.0 \\
\mathrm{f}_{\mathrm{v}, \text { max }} & =8.4 \mathrm{ppm} \\
\mathrm{z}_{\text {max }} & =13.9 \mathrm{~mm} \\
\mathrm{~T}_{\text {max }} & =2334 \mathrm{~K}
\end{aligned}
$$

$$
\overline{f_{v, \max }} \Delta\left(\frac{f_{v}}{f_{v, \max }}\right)
$$

$$
\mathrm{r} / \mathrm{d}_{\mathrm{F}}
$$

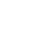


LaTex Source File
Click here to down

LaTex Source File
Click here to download LaTex Source File: Jocheretal.tex

(1)

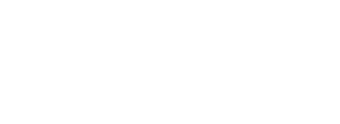

.

(1)

- n

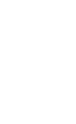

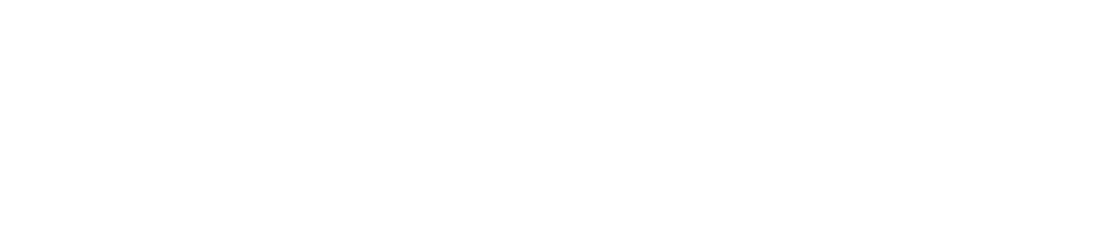

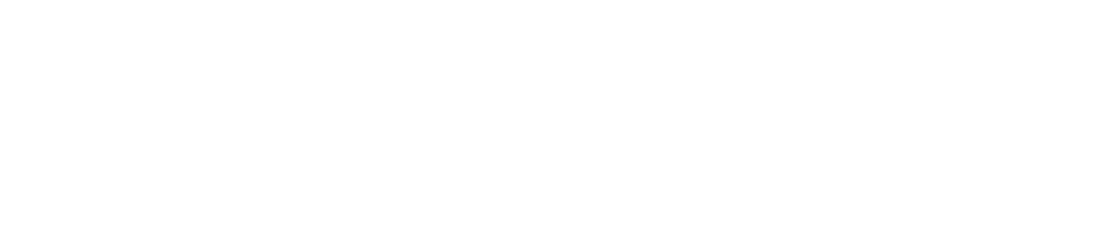

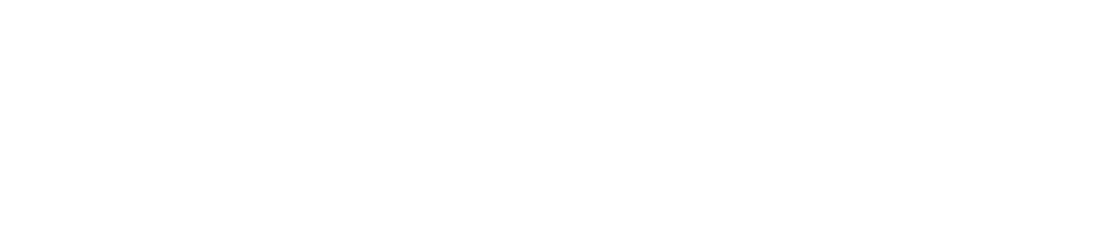

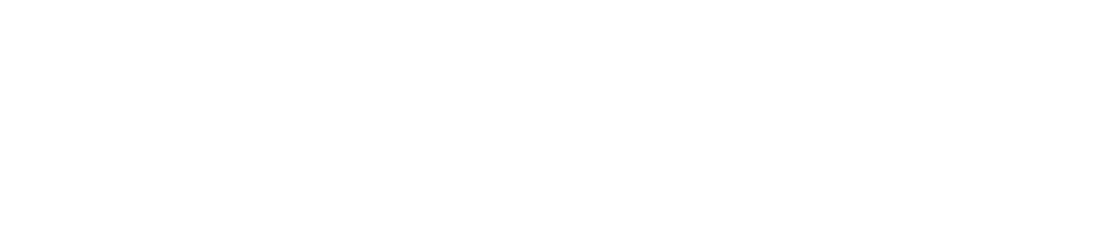

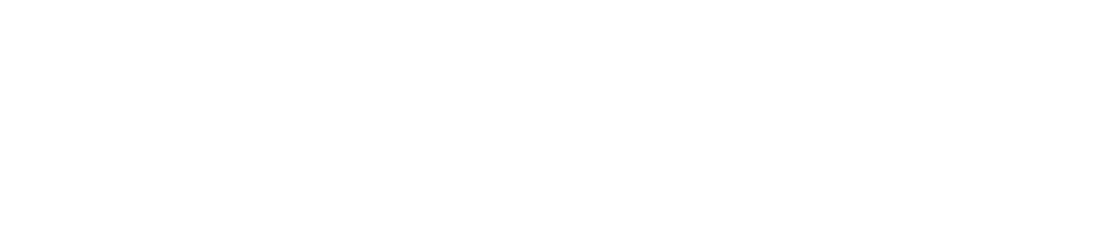

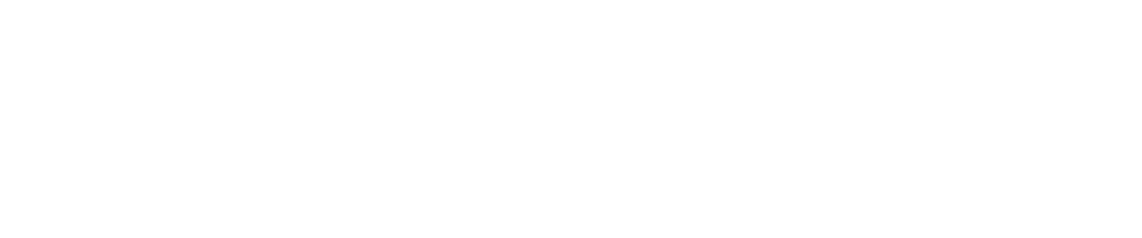

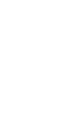

.

(1) 